\title{
A CLASS OF UNIFORMLY HARMONIC FUNCTIONS OF TYPE K USING SALAGEAN OPERATORS
}

\author{
DR .NOOHI KHAN (AP II)
}

Amity University Lucknow, Uttar Pradesh, India

\begin{abstract}
This paper introduces a new generalized function $\operatorname{USHK}(\mu, v, \alpha)$ which is a sub class of $k$-uniformly harmonic

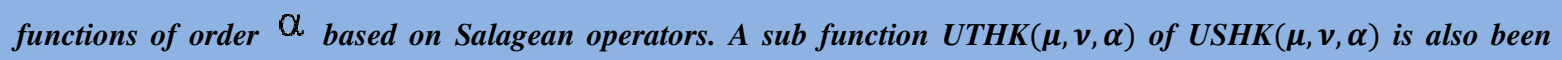
defined and studied in this Paper.

KEYWORDS: Harmonic, Univalent, Salagean, Generalized
\end{abstract}

Received: Jun 27, 2020; Accepted: Jul 17, 2019; Published: Sep 22, 2020; Paper Id.: IJMCARDEC20205

\section{INTRODUCTION}

Suppose the class SH indicates the class of functions $F=H+\bar{G}$ which are defined in the open disk $D=\{Z:|Z|<$ 1 ) of radius unity for which the normalization condition holds $F(0)=F(0)-1=0$. The function $\mathrm{F}$ is univalent as well as harmonic and also $\mathrm{F}$ preserves the orientation and then for $F=H+\bar{G} \in S H$ the analytic functions $\mathrm{H}$ and $\mathrm{G}$ is defined as

(1a) $\quad H(Z)=Z+\sum_{N=2}^{\infty} A_{N} Z^{N}, G(Z)=\sum_{N=1}^{\infty} B_{N} Z^{N},\left|B_{1}\right|<1$.

Where $\mathrm{H}$ denotes analytic portion and $\mathrm{G}$ denotes the co analytic portion of F. Salagean [7] defined an operator given below:

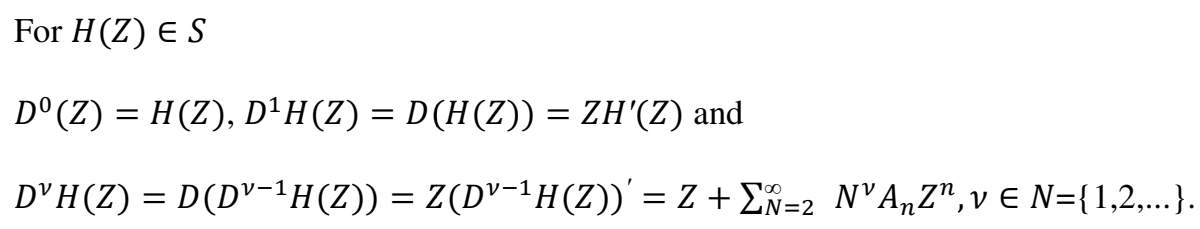

Where D is called the Salagean operator.

Jahangiri et al. [9,10] introduced the modified Salagean operator of harmonic functions $\mathrm{F}$ which is univalent, also as given

$$
D^{v} F(Z)=D^{v} H(Z)+\underline{(-1)^{v} D^{v} G(Z)}, \quad v \in \mathbf{N}_{0}=\{0,1,2, \ldots\}
$$

where $D^{v} H(Z)=Z+\sum_{N=2}^{\infty} N^{v} A_{N} Z^{N}$ and $D^{v} G(Z)=\sum_{N=1}^{\infty} N^{v} B_{N} Z^{N}$.

Definition: For $0 \leq \alpha<1,0 \leq k<\infty, \mu>v$, $\operatorname{USHK}(\mu, v, \alpha)$ is the class in which function $F=H+G$ is defined and satisfying the following condition: 
(1b ) $\left\{\left(1+k e^{i \varphi}\right) \frac{D^{\mu} F(z)}{D^{v} F(z)}-k e^{i \varphi}\right\} \geq \alpha, \phi \in \mathbf{R}$

Also, $\operatorname{UTHK}(\mu, v, \alpha) \subseteq \operatorname{USHK}(\mu, v, \alpha)$ is the class of harmonic functions $F_{\mu}=H+\bar{G}_{\mu}$ which are of negative coefficients so that

(1c) $\quad H(Z)=Z-\sum_{N=2}^{\infty}\left|A_{N}\right| Z^{n}, G(Z)=(-1)^{\mu-1} \sum_{N=1}^{\infty}\left|B_{N}\right| Z^{N},\left|B_{1}\right|<1$.

Also $\operatorname{USHK}(1,0, \alpha) \equiv \operatorname{USHK}(\alpha)$,

$\operatorname{USHK}(2,1, \alpha) \equiv \operatorname{UCHK}(\alpha)$ and classes $\operatorname{UTHK}(\alpha) \subseteq U S H K(\alpha)$ and $\operatorname{THCVK}(\alpha) \subseteq U C H K(\alpha)$ consist of functions $\mathrm{F}$ of the form (3).

Also $\quad 1-U S H(0) \equiv G_{H}, 1-H C V(0) \equiv H C V$.

Generalization: The class UTHK $(\mu, v, \alpha)$ generalizes several groups of harmonic functions which are univalent and also are defined earlier.

1) For $\mathrm{k}=0, \mu=\mathbf{1}, v=0$, this class reduces to $\mathrm{SH}(\alpha)$ of order $\alpha$ which was studied by Jahangiri [9].

2) For $k=0, \mu=2, v=1$, it reduces to the class $K H(\alpha)$, of order $\alpha$ which was studied by Jahangiri [10].

3) For k=1, $\boldsymbol{\mu}=\mathbf{1}, v=\mathbf{0}$, this class reduces to $\mathbf{G}_{\mathbf{H}}(\boldsymbol{\alpha})$ which was studied by Rosy et al. [11].

4) For $\mathrm{k}=1, \mu=v+1$, this class reduces to $\operatorname{RS}_{H}(v, \alpha)$ which was studied by Yalcin et al. [12].

\section{SUFFICIENT COEFFICIENT CONDITION FOR USHK $(\mu, v, \alpha)$}

Theorem 2.1: The function $F$ be given by (1a). Furthermore, let

$$
\sum_{n=1}^{\infty}\left\{\psi(\mu, v, \alpha)\left|A_{n}\right|+\theta(\mu, v, \alpha)\left|B_{n}\right|\right\} \leq 2
$$

where, $\quad \psi(\mu, v, \alpha)=N^{v}+\frac{\left(N^{\mu}-N^{v}\right)(1+k)}{1-\alpha}$

$$
\theta(\mu, v, \alpha)=(-1)^{v-\mu} N^{v}+\frac{\left(N^{\mu}-(-1)^{\nu-\mu} N^{v}\right)(1+k)}{1-\alpha}
$$

with $A_{1}=1,0 \leq \alpha<1,0 \leq k<\infty, \mu \in N=\{1,2, \ldots\}, v \in N_{o}$ and $\mu>v$, then $\mathrm{F}$ is univalent, harmonic and preserve the orientation in $\mathrm{D}$ and $\operatorname{F\in USHK}(\mu, v, \alpha)$. The result is sharp also.

\section{Proof}

For $|Z| \leq\left|Z_{2}\right|<1$ preserving in $\mathrm{D}$, this is because

$$
\begin{aligned}
& \left|H^{\prime}(z)\right| \geq 1-\sum_{N=2}^{\infty} N\left|A_{N}\right||Z|^{N-1} \\
& \geq 1-\sum_{N=2}^{\infty} N\left|A_{N}\right| \\
& \geq 1-\sum_{N=2}^{\infty}\left[N^{v}+\frac{\left(N^{\mu}-N^{v}\right)(1+k)}{1-\alpha}\right]\left|A_{N}\right| \\
& \geq \alpha+\sum_{N=1}^{\infty}\left[(-1)^{v-\mu} N^{v}+\frac{\left(N^{\mu}-(-1)^{v-\mu} N^{v}\right)(1+k)}{1-\alpha}\right]\left|B_{N}\right| \text { (using 2) }
\end{aligned}
$$




$$
\begin{aligned}
& \geq \sum_{N=1}^{\infty}\left[(-1)^{\nu-\mu} N^{\nu}+\left(N^{\mu}-(-1)^{\nu-\mu} N^{\nu}\right)(1+k)\right]\left|B_{N}\right| \\
& \qquad \sum_{N=1}^{\infty} N\left|B_{N}\right| \\
& \geq \sum_{N=1}^{\infty} N\left|B_{N}\right||Z|^{N-1} \\
& \geq\left|G^{\prime}(Z)\right| .
\end{aligned}
$$

It only needs to show that $\mathrm{F} \in \operatorname{USHK}(\mu, v, \alpha)$

That is

$$
\left\{\frac{\left(1+k e^{i \varphi}\right) D^{\mu} F(Z)-\left(k e^{i \varphi}+\alpha\right) D^{v} F(Z)}{D^{v} F(Z)}\right\} \geq 0
$$

or,

$\left\{\frac{\left(Z+\sum_{N=2}^{\infty} N^{\mu} A_{N} Z^{N}+(-1)^{\mu} \sum_{N=1}^{\infty} N^{\mu} B_{N} \bar{Z}^{N}\right)\left(1+k e^{i \phi}\right)-\left(k e^{i \phi}+\alpha\right)\left(Z+\sum_{N=2}^{\infty} N^{v} A_{N} Z^{N}+(-1)^{v} \sum_{N=1}^{\infty} N^{v} \bar{B}_{N} \bar{Z}^{N}\right)}{Z+\sum_{N=2}^{\infty} N^{v} A_{N} Z^{N}+(-1)^{v} \sum_{N=1}^{\infty} N^{v} \bar{B}_{N} N}\right\}$ For $Z=R e^{i \theta}$,

$$
\begin{gathered}
A\left(R e^{i \theta}\right)=\sum_{N=2}^{\infty}\left[N^{\mu}\left(1+k e^{i \phi}\right)-N^{\nu}\left(k e^{i \phi}+\alpha\right)\right] A_{N} R^{N-1} e^{(N-1) \theta i}++(-1)^{\mu} \sum_{N=1}^{\infty}\left[N^{\mu}\left(1+k e^{i \phi}\right)-(-1)^{\nu-\mu} N^{\nu}\left(k e^{i \phi}\right.\right. \\
+\alpha) \bar{B}_{N} R^{N-1} e^{-(N+1) \theta i}
\end{gathered}
$$

$B\left(R e^{i \theta}\right)=\sum_{N=2}^{\infty} N^{v} A_{N} R^{N-1} e^{(N-1) \theta i}+(-1)^{v} \sum_{N=1}^{\infty} N^{v} B_{N} R^{N-1} e^{-(N+1) \theta i}$.

Setting $\frac{(1-\alpha)+A(Z)}{1+B(Z)}=(1-\alpha) \frac{1+w(Z)}{1-w(Z)}$

The proof will be completed if it can show $|w(Z)| \leq R<1$.

Since,

$$
\begin{aligned}
& |w(Z)|=\left|\frac{A(Z)-(1-\alpha) B(Z)}{A(Z)+(1-\alpha) B(Z)+2(1-\alpha)}\right| \\
& =\left|\begin{array}{l}
\sum_{N=2}^{\infty}\left[N^{\mu}\left(1+k e^{i \varphi}\right)-N^{\nu}\left(k e^{i \varphi}+\alpha\right)-(1-\alpha) N^{\nu}\right] A_{N} R^{N-1} e^{(N-1) \theta i} \\
+(-1)^{\mu} \sum_{N=1}^{\infty}\left[N^{\mu}\left(1+k e^{i \varphi}\right)-(-1)^{v-\mu}\left(k e^{i \varphi}+\alpha\right) N^{\nu}-(1-\alpha)(-1)^{\nu-\mu} N^{v}\right] \overline{B_{n}} R^{N-1} e^{-(N+1) \theta i} \\
\hline 2(1-\alpha)+\sum_{N=2}^{\infty}\left[N^{\mu}\left(1+k e^{i \varphi}\right)-N^{v}\left(k e^{i \varphi}+\alpha\right)+(1+\alpha) N^{v}\right] A_{N} R^{N-1} e^{(N-1) \theta i} \\
+(-1)^{\mu} \sum_{N=1}^{\infty}\left[N^{\mu}\left(1+k e^{i \varphi}\right)-(-1)^{-\mu+v} N^{\nu}\left(k e^{i \varphi}+\alpha\right)+(-1)^{\nu-\mu} N^{\nu}(1-\alpha)\right] \bar{B}_{N} R^{N-1} e^{-(N+1) \theta i}
\end{array}\right| \\
& \leq \frac{\left.\sum_{N=2}^{\infty}\left[N^{\mu}-N^{v}\right)(1+k)\right]\left|A_{N}\right| R^{N-1}+\sum_{N=1}^{\infty}\left[\left(N^{\mu}-(-1)^{\nu-\mu} N^{v}\right)(1+k)\right]\left|B_{N}\right| R^{N-1}}{2(1-\alpha)-\sum_{N=2}^{\infty}\left[\left(N^{\mu}-N^{v}\right) k+N^{\mu}-2 \alpha N^{v}+N^{v}\right]\left|A_{N}\right| R^{N-1}} \\
& -\sum_{N=1}^{\infty}\left[\left(N^{\mu}-(-1)^{\nu-\mu} N^{v}\right) k+N^{\mu}-2 \alpha(-1)^{v-\mu} N^{v}\right. \\
& \left.+(-1)^{\nu-\mu} N^{v}\right]\left|B_{N}\right| R^{N-1}
\end{aligned}
$$

where, $\quad C(\mu, v, \alpha)=\left(N^{\mu}-N^{v}\right) k+N^{\mu}+(1-2 \alpha) N^{v}$

$$
\begin{aligned}
D(\mu, v, \alpha)= & \left(N^{\mu}-(-1)^{v-\mu} N^{v}\right) k+N^{\mu}+(1-2 \alpha)(-1)^{v-\mu} N^{v} \\
& =\frac{\sum_{N=2}^{\infty}\left[\left(N^{\mu}-N^{v}\right)(1+k)\right]\left|A_{N}\right|+\sum_{N=1}^{\infty}\left[\left(N^{\mu}-(-1)^{v-\mu} N^{v}\right)(1+k)\right]\left|B_{N}\right|}{4(1-\alpha)-\sum_{N=1}^{\infty}\left\{C(\mu, v, \alpha)\left|A_{N}\right|+D(\mu, v, \alpha)\left|B_{N}\right|\right\}}
\end{aligned}
$$

$\leq 1$ (using 2a). 
which proves the Theorem.

\section{Sharpness of the Result}

The function:

$$
F(Z)=Z+\sum_{N=2}^{\infty} \frac{1}{\psi(\mu, v, \alpha)} X_{N} Z^{N}+\sum_{N=1}^{\infty} \frac{1}{\theta(\mu, v, \alpha)} Y_{N} \bar{Z}^{N}
$$

where $\mu \in N, v \in N_{0}, \mu>v$ and $\sum_{N=2}^{\infty}\left|X_{N}\right|+\sum_{N=1}^{\infty}\left|Y_{N}\right|=1$, shows that the coefficient bound given by (2a) is sharp. The functions of the form $(2 \mathrm{a})$ are in $\operatorname{USHK}(\mu, v, \alpha)$ because

$$
\sum_{N=1}^{\infty}\left\{\psi(\mu, v, \alpha)\left|A_{N}\right|+\theta(\mu, v, \alpha)\left|B_{N}\right|\right\}=1+\sum_{N=2}^{\infty}\left|X_{N}\right|+\sum_{n=1}^{\infty}\left|Y_{N}\right|=2 .
$$

\section{REFERENCES}

1. Ahuja, O.P., Jahangiri, J.M., and Silverman,H., Convolutions for special classes of Harmonic Univalent Functions, Appl. Math. Lett, 16(6) (2003), 905-909.

2. Ahuja,O.P. and Silverman,H., Extreme Points of families of Univalent Functions with fixed second coefficient. Colloq. Math. 54 (1987), 127-137.

3. Ahuja,O.P. and Jahangiri,J.M., Harmonic Univalent Functions with fixed second coefficient, Hakkoido Mathematical Journal, Vol. 31(2002) p-431-439.

4. Ahuja,O.P. and Silverman,H., Inequalities associating hypergeometric Functions with planar harmonic mappings, Vol 5, Issue 4, Article 99, 2004.

5. Ahuja,O.P. and Jahangiri,J.M., Multivalent Harmonic starlike Functions, Ann. Univ Mariae Curie - Sklodowska, Section A, 55 (1) (2001), 1-13.

6. Ahuja,O.P. and Jahangiri,J.M., A subclass of Harmonic Univalent Functions, J.Natural geometry, 20, (2001), 45-56.

7. Salagean, G.S., Subclass of Univalent Functions, Lecture Notes in Math. Springer - Verlag 1013(1983), 362-372.

8. Jahangiri,J.M., Murugusundarmoorthy,G. and Vijaya,K., Salagean type Harmonic Univalent Functions, South. J. Pure and Appl. Math., Issue 2(2002), 77-82.

9. Jahangiri,J.M., Harmonic Functions starlike in the unit disc, J. Math. Anal. Appl., 235 (2) (1999), 470-477.

10. Jahangiri,J.M., Coefficient bounds and univalence criteria for harmonic functions with negative coefficient, Ann. Univ. Mariae, Curie-Skledowska Sect. A, 52(2) (1998), 57-66.

11. Rosy,T., Adolph Stephen,B. and Subramanian,K.G., Goodman Ronning type Harmonic Univalent Functions, Kyungpook Math., J., 41(2001), 45-54.

12. Yalcin,S., zt rk,M. and Yamankaradeniz,M., On the subclass of Salagean type Harmonic Univalent Functions

13. K. Noohi; A subclass of harmonic univalent functions, compliance Engineering Journal Vol10, Issue 9, (2019) 373 - 379. 